\title{
Diferencias entre adolescentes del área rural-urbana en malos tratos durante el noviazgo*
}

\section{Differences between rural and urban adolescents in dating violence}

\author{
César Armando Rey Anacona** \\ ORCID 0000-0002-9528-2199 \\ Universidad Pedagógica y Tecnológica de \\ Colombia \\ Jorge Arturo Martínez Gómez, \\ ORCID 0000-0002-1423-3812 \\ Universidad Pedagógica y Tecnológica de \\ Colombia

\section{Nora Helena Londoño Arredondo ORCID 0000-0001-6016-1955 \\ Universidad San Buenaventura Sede Medellín}

Recibido: 26 de abril de 2016 Revisado: 20 de mayo de 2017 Aceptado: 3 de junio de 2017

\section{Resumen}

Se examinaron las diferencias existentes en las conductas de maltrato ejercidas en el noviazgo en adolescentes de un área rural y urbana del municipio de Tunja (Colombia), mediante un diseño trasversal descriptivo-comparativo, en el que participaron 119 mujeres y 113 varones de 13 a 19 años de dos colegios públicos, uno urbano $(n=152)$ y otro rural $(n=80)$. Para ello se utilizó una lista de chequeo de experiencias de maltrato de pareja. Los resultados señalan que aproximadamente la mitad ejecutó al menos una conducta de este tipo, tanto en la zona urbana como rural, aunque la frecuencia de comportamientos de maltrato fue significativamente mayor entre los(as) del área urbana, a nivel general y en los tipos de maltrato psicológico, emocional y físico. Estos resultados señalan que estas conductas podrían ser más frecuentes entre los adolescentes de áreas urbanas, aunque se requiere más investigación y atención sobre este fenómeno en zonas rurales.

Palabras clave: violencia en el noviazgo, adolescentes, rural, urbano, estudiantes. 


\section{Abstract}

We examined the differences in dating violence in adolescents in a rural and urban area of the municipality of Tunja (Colombia), through a descriptive-comparative cross-sectional design, in which 119 women and 113 men participated, aged 13 to 19 years of two public schools, one urban $(n=152)$ and one rural $(n=80)$. For this, a checklist of partner abuse experiences was used. The results indicate that approximately half performed at least one behavior of this type, both in the urban and rural areas, although the frequency of maltreatment behaviors was significantly higher among those in the urban area, in general, and in the types of psychological, emotional and physical abuse. These results indicate that these behaviors may be more frequent among adolescents in urban areas, although more research and attention is required to focus on rural areas.

Keywords: dating violence, adolescents, rural, urban, students.

El fenómeno de los malos tratos en parejas jóvenes ha despertado un amplio interés entre los investigadores de diferentes partes del mundo, no solo por sus posibles secuelas en las víctimas sino por su papel en la predicción de la violencia en la vida adulta (Arbach, Nguyen-Vo \& Bobbio, 2015; González-Ortega, Echeburúa y Corral, 2008). Las cifras de prevalencia indican que al menos la mitad de los encuestados han sido objeto de alguna forma de maltrato por parte de su pareja (Garrido \& Taussig, 2013; Muñoz, Ortega-Rivera y Sánchez, 2013).

En Iberoamérica, en particular, dicho interés se ha centrado en la prevalencia del fenómeno (e.g., Cortés-Ayala, Flóres, Bringas, Rodríguez-Franco, López-Cepero y Rodríguez-Díaz, 2015; MuñozRivas, Graña, O’Leary y González, 2007; Rivera, Allen, Rodríguez, Chávez y Lazcano, 2007; ReyAnacona, 2013; Vivanco, Espinoza, Romo, Véliz y Vargas, 2015), sus posibles factores de riesgo y dificultades asociadas (e.g. Borrajo, Gámez-Guadix y Calvete, 2015; Martínez, Vargas y Novoa, 2016; Muñoz-Rivas, Gámez-Guadix, Graña y Fernández, 2010; Muñoz-Rivas et al., 2007; Rey-Anacona, 2011; Rey-Anacona, 2015; Saldivia y Vizcarra, 2012) y en el diseño y evaluación de programas de prevención (e.g., Hernando, 2007; Muñoz et al., 2013; Pick, Leenen, Givaudan y Prado, 2010; Póo y Vizcarra, 2011). Sin embargo, ninguno de estos estudios discriminó las muestras en cuanto a su procedencia rural o urbana, por lo que aún no se tiene una aproximación a la magnitud del fenómeno en las zonas rurales y las variables que podrían estar asociadas con la realización de este tipo de conductas.

Desde los años noventa, en los Estados Unidos se han realizado algunos estudios de prevalencia de malos tratos en parejas jóvenes, teniendo en cuenta su procedencia, rural o urbana. Bergman (1992) comparó la prevalencia de victimización entre 631 estudiantes de secundaria, varones y mujeres de comunidades rurales, sub-urbanas y urbanas, y encontró que era mayor el porcentaje de mujeres que reportaron haber recibido violencia física y sexual, comparado con el de los varones $(24,6 \%$ versus $9,9 \%$ ). El porcentaje de adolescentes de áreas urbanas que ejercieron conductas de maltrato a su pareja fue visiblemente mayor $(54,1 \%)$, al de los adolescentes de áreas sub-urbanas $(29,1 \%$ ) y rurales (19,3\%). Spencer y Bryant (2002) realizaron un estudio similar con 2.094 estudiantes de séptimo a undécimo grado, de comunidades urbanas, suburbanas y rurales estadounidenses, y encontró que los varones fueron objeto de una frecuencia mayor de violencia física que las mujeres, así como los participantes de las comunidades rurales, comparados con los de las áreas suburbanas y urbanas, es decir, sus resultados fueron opuestos a los de Bergman.

Marquart, Nannini, Edwards, Stanley y Wayman (2007), por su parte, hallaron en una muestra nacional conformada por 20.274 adolescentes que vivían en áreas rurales, que el 15,8\% había sido objeto de malos tratos por parte de su pareja, siendo esta prevalencia mayor entre las mujeres y entre los adolescentes del sur del país. 
Otros estudios realizados solamente con participantes de comunidades rurales o mayoritariamente rurales estadounidenses se han enfocado en la relación entre la violencia con diferentes dificultades de salud física y mental. Así, Foshee, McNaughton, Gottfredson, Chang y Ennett (2013) realizaron un estudio longitudinal con 3.328 estudiantes de dos condados del Estado de Carolina del Norte, de grados octavo a duodécimo, y encontraron que el ser víctima de violencia psicológica era predicho por un consumo incrementado de bebidas alcohólicas, mientras que la violencia física era predicha por el consumo de cigarrillo. McDonell, Ott y Mitchell (2010), por su parte, examinaron un conjunto de variables que podrían estar relacionadas con la victimización y la perpetración entre 351 estudiantes de sexto a duodécimo grados de una comunidad rural de Carolina del Sur, lo que evidenció que la victimización entre las mujeres y los varones, así como la perpetración femenina, se relacionaban con el uso de sustancias y las actitudes a favor de la violencia, mientras que la perpetración por parte de los varones se asociaba con el involucramiento en el sistema de justicia juvenil, las actitudes a favor de la violencia y la aceptación de comportamientos relacionados con la violencia en la familia y en las relaciones de noviazgo. Montoya, Coker-Appiah, Eng, Wynn y Townsend (2013), realizaron un estudio con una metodología cualitativa sobre el efecto percibido de la violencia en el noviazgo sobre la salud, en un grupo de 20 afroamericanos de áreas rurales, quienes percibieron efectos negativos de este tipo de violencia sobre la conducta sexual.

Estos datos sugieren que muchos adolescentes de comunidades rurales ejercen o han sido víctimas de malos tratos por parte de su pareja y que esta podría estar relacionada con variables similares a las que se han encontrado en adolescentes reclutados en sectores urbanos, como el uso de sustancias y las actitudes a favor de la violencia (e.g., MuñozRivas et al., 2010; Rey-Anacona, 2015; Sears, Byers \& Price, 2007). Sin embargo, los estudios aún son escasos y con resultados contradictorios.

En Colombia algunos estudios indican cifras elevadas de adolescentes y adultos jóvenes involucrados en relaciones afectivas en las que se ha presentado alguna forma de maltrato (Vg., Martínez et al.,
2016; Rey-Anacona, 2009; Rey-Anacona, 2013). Sin embargo, ninguno de estos estudios discriminó la procedencia rural o urbana de los participantes, a pesar de que la población que vive en comunidades rurales en dicho país alcanza el $24 \%$ (Banco Mundial, 2016). Según el Tercer Censo Nacional Agropecuario realizado en 2014, cuya cobertura alcanzó al 98,9\% de los municipios de Colombia, el $44,7 \%$ de la población rural colombiana se encuentra bajo el límite de pobreza, según el índice de pobreza multidimensional; es decir, casi el triple del registrado en la población urbana, en donde la pobreza alcanza al 15,4\%. Aunque la mayoría cuenta con cobertura en el sistema de salud, no tiene la infraestructura agropecuaria necesaria para la producción tecnificada; además, el 11,5\% de la población rural mayor de 15 años no sabe leer ni escribir y el $76 \%$ de los habitantes entre 17 y 24 años de edad se encuentran desvinculados del sistema educativo (Departamento Administrativo Nacional de Estadística, 2015a).

En el departamento de Boyacá, en particular, aproximadamente la mitad de la población vive en zonas rurales (Gobernación de Boyacá, 2011). El porcentaje de personas en situación de pobreza en 2014 alcanzaba el 38,2 \% (Departamento Administrativo Nacional de Estadística, 2015b). Su cultura y composición racial evidencia una mezcla de las costumbres aborígenes y españolas, introducidas durante el período colonial, con una fuerte influencia del catolicismo (Díaz, 2012).

Debido a estas diferencias sociales y demográficas, es posible que el fenómeno de la violencia en el noviazgo en la adolescencia se manifieste de forma diferente en los sectores rurales colombianos con respecto a los sectores urbanos, lo cual podría tener implicaciones en la comprensión y abordaje del fenómeno a nivel de evaluación, prevención y tratamiento.

Conforme a lo anterior, este estudio se planteó como objetivo describir y determinar las diferencias existentes en las conductas de maltrato ejercidas en el noviazgo en adolescentes de un área rural y urbana del Municipio de Tunja, en el Departamento de Boyacá, Colombia, al examinar examinando las diferencias existentes por sexo. 


\section{Método}

\section{Diseño}

Se llevó a cabo un estudio de corte observacional, trasversal descriptivo y comparativo (Méndez y Nahimira, 2000), con información suministrada por una muestra no probabilística por conveniencia.

\section{Participantes}

El grupo de estudio estuvo conformado por 232 estudiantes que informaron tener una pareja heterosexual (51,3 \% mujeres y $48,7 \%$ hombres). Los participantes se encontraban cursando los grados noveno $(28 \%)$, decimo $(39,2 \%)$ y undécimo $(32,8 \%)$ en dos colegios públicos, uno de la zona urbana (152 participantes) y otro rural (80 participantes), con edades comprendidas entre los 13 y los 19 años $(M=15,26 ; D T=1,22)$. El nivel socioeconómico se distribuyó en el estrato uno $(57,8 \%)$, estrato dos $(32,8 \%)$ y estrato tres $(9,5 \%)$. El $44,8 \%$ de los padres de los participantes estaban casados, el $33,2 \%$ en unión libre, el 17,2 \% separados y un $4,7 \%$ divorciados, viudos o en concubinato. Para la participación en la investigación, todos los encuestados debían reportar tener o haber tenido uno o más relaciones previas a la investigación con una duración mínima de tres meses, contar con la firma del consentimiento informado por parte de los padres, si se trataba de menores de edad y la aceptación voluntaria de participar en el estudio.

\section{Instrumento}

Lista de chequeo de experiencias de maltrato en la pareja forma B (Rey-Anacona, MateusCubides y Bayona-Arévalo, 2010). Permite reportar la realización de 82 conductas de maltrato hacia la pareja, de tipo físico, emocional, psicológico, sexual, económico y negligente, a través de una escala tipo Likert con cuatro opciones de respuesta ("Nunca": 0, "Una vez": 1; "Algunas veces": 2 y "Muchas veces": 4). El instrumento presentó un alfa de 0,96 en su puntuación total en una muestra constituida por 562 adolescentes entre 15 y 20 años, lo que evidenció que el 50,54 \% de la varianza podría explicarse por seis factores (Rey-Anacona et al., 2010). En esta investigación se examinó la frecuencia general y de cada uno de estos tipos de maltrato que reportó haber ejercido cada participante, excepto los de tipo económico y negligente debido a que son más comunes en parejas casadas o en convivencia que en adolescentes.

En este instrumento, cada tipo de maltrato se define de la siguiente manera (Rey-Anacona et al., 2010): (a) maltrato físico: actos dirigidos al cuerpo de la persona, que podrían producir daño o dolor sobre ella; (b) maltrato psicológico: acciones que buscan controlar, restringir los movimientos o vigilar a la otra persona, aislarla socialmente, desvalorizarla o hacerla sentir mal consigo misma, poner a otros en contra suyo, acusarla falsamente o culparla por circunstancias negativas, obligarla a ir en contra de la ley o de sus creencias morales y/o religiosas o destruir su confianza en sí misma o en la pareja; (c) maltrato emocional: conductas verbales y no verbales que pueden provocar en la víctima una reacción de ansiedad, temor o miedo, como las amenazas y los actos de violencia dirigidos a un familiar o a un conocido de la víctima, a sus bienes o hacia el agresor mismo y (d) maltrato sexual: actos no consentidos por la víctima dirigidos a satisfacer necesidades o deseos sexuales del victimario.

\section{Procedimiento}

Después de obtener el permiso de las instituciones educativas correspondientes, así como el consentimiento escrito por parte de los adolescentes y sus padres (excepto los mayores de edad), la información se recolectó mediante aplicaciones grupales en los salones de clase de los participantes. Los instrumentos fueron administrados por un psicólogo, con una duración aproximada de 35 minutos. La participación fue voluntaria y anónima, no remunerada. Se utilizó una ficha sociodemográfica para recoger la siguiente información de los participantes: edad, sexo, estrato socioeconómico, el grado que cursaban, zona de vivienda (rural-urbano) y estructura familiar. 


\section{Análisis estadísticos}

Los análisis estadísticos se realizaron con el programa SPSS versión 21,0 . Se llevaron a cabo análisis descriptivos y comparativos en relación con el número de participantes y la frecuencia de conductas de violencia ejercidas, tanto a nivel general como por cada tipo (psicológica, física, emocional, sexual, económica), así como comparaciones por sexo según la zona de procedencia. Para efectuar estas comparaciones se utilizaron las pruebas no paramétricas Chi-Cuadrado, U de Mann-Whitney y Kruskal-Wallis, debido a que la prueba de normalidad Shapiro-Wilk, mostró que los datos de los seis tipos de violencia no se ajustaban normalmente entre los grupos del área rural y urbana $(p \leq 0,05)$.

\section{Consideraciones éticas}

Con base a la Resolución 008430 de 1993 del Ministerio de Salud de Colombia, la investigación tenía un nivel de riesgo mínimo; se solicitó el consentimiento informado de manera escrita a los participantes y sus padres si eran menores de edad, donde se les informó la relevancia social y científica del trabajo, su objetivo, procedimiento, confidencialidad y anonimato, explicándose que la participación no tendría ninguna consecuencia. Dentro de los principios éticos se tuvo en cuenta: la responsabilidad, derecho a la no-participación, respeto, confidencialidad de la información recogida y derecho a ser informado de los resultados, además de evitar el uso indebido de los datos recolectados, conforme a la Ley 1090 del 6 de septiembre de 2006, que reglamenta el ejercicio de la psicología en Colombia.

\section{Resultados}

El 48,7\% de los participantes informó haber presentado una conducta de maltrato hacia su pareja al menos una vez, $42.5 \%$ de la zona rural y $52 \%$ de la zona urbana, en lo que nos se encontró no encontrándose una diferencia estadísticamente significativas entre estos dos porcentajes: $X^{2}(1, N=232)$ $=1,883, p=0,170$. Con respecto al maltrato psicológico $44,8 \%$ de los estudiantes informaron haber utilizado este tipo de conductas en su relación de noviazgo al menos una vez, $38,8 \%$ de los estudiantes de la zona rural y $48 \%$ de los estudiantes de la zona urbana. El 15,5\% de los adolescentes reportaron el uso de maltrato emocional en su relación de pareja al menos en una ocasión, 11,3 \% de los estudiantes de la zona rural y $17,8 \%$ de los estudiantes de la zona urbana. De igual forma, un 15,5\% informó haber utilizado conductas de maltrato físico por lo menos en una oportunidad, $11,3 \%$ de los estudiantes de la zona rural y $17,8 \%$ de los estudiantes de la zona urbana. Con respecto a conductas de maltrato sexual, el 10,8 \% informó haber utilizado este tipo de conductas al menos en una ocasión, 11,3\% de los estudiantes de la zona rural y $10,5 \%$ de los estudiantes de la zona urbana.

Tabla 1.

Diferencias (U de Mann-Whitney), en el rango promedio de comportamientos de maltrato según la zona de procedencia de los participantes (rural-urbana).

\begin{tabular}{|c|c|c|c|c|c|c|}
\hline Tipo de maltrato & Zona & $n$ & Mediana & Rango promedio & $z$ & $p$ \\
\hline \multirow{2}{*}{ Psicológico } & Rural & 80 & 3,34 & 104.30 & \multirow{2}{*}{$-2,073$} & \multirow{2}{*}{$0,038^{*}$} \\
\hline & Urbano & 152 & 8,19 & 121.47 & & \\
\hline \multirow{2}{*}{ Emocional } & Rural & 80 & 0,31 & 107.98 & \multirow{2}{*}{$-2,283$} & \multirow{2}{*}{$0,022^{*}$} \\
\hline & Urbano & 152 & 1,52 & 120.99 & & \\
\hline \multirow{2}{*}{ Físico } & Rural & 80 & 0,44 & 108.52 & \multirow{2}{*}{$-2,063$} & \multirow{2}{*}{$0,039^{*}$} \\
\hline & Urbano & 152 & 1,58 & 119.96 & & \\
\hline \multirow{2}{*}{ Sexual } & Rural & 80 & 0,44 & 116.11 & \multirow{2}{*}{$-0,121$} & \multirow{2}{*}{0,904} \\
\hline & Urbano & 152 & 0,51 & 116.70 & & \\
\hline \multirow{2}{*}{ General } & Rural & 80 & 4,75 & 105.07 & \multirow{2}{*}{$-2,081$} & \multirow{2}{*}{$0,048^{*}$} \\
\hline & Urbano & 152 & 12,26 & 121.06 & & \\
\hline
\end{tabular}

${ }^{*} p \leq 0,05$ 
Como se presenta en la Tabla 1, los participantes de la zona urbana reportaron un rango promedio de comportamientos de maltrato psicológico, emocional y físico, significativamente mayor que los participantes de la zona rural, así como en la frecuencia general de malos tratos.

En la tabla 2 se presentan los ítems de la Lista de chequeo de experiencias de maltrato en la pareja forma B (Rey-Anacona et al., 2010), en donde se presentaron diferencias estadísticamente significativas entre los dos grupos de participantes, con un rango promedio estadísticamente mayor entre los estudiantes de la zona urbana comparados con los del área rural.

Finalmente, en la tabla 3, se presentan las comparaciones efectuadas por sexo y zona, en donde se evidencia que los hombres del área urbana presentaron un rango promedio significativamente mayor de conductas de maltrato psicológico, emocional y sexual, así como a nivel general. No se halló una diferencia estadísticamente significativa en cuanto a las conductas de violencia física.

Tabla 2.

Diferencias en comportamientos específicos de maltrato entre los dos grupos de participantes (U de Mann-Whitney)

\begin{tabular}{|c|c|c|c|c|c|c|}
\hline Ítems & Zona & $n$ & Mediana & $\begin{array}{c}\text { Rango } \\
\text { promedio }\end{array}$ & $Z$ & $p$ \\
\hline \multirow{2}{*}{ 19. Rompió o destruyó algún bien personal suyo. } & Rural & 80 & 0,03 & 110,44 & \multirow{2}{*}{$-2,343$} & \multirow{2}{*}{$0,019^{*}$} \\
\hline & Urbana & 152 & 0,21 & 119,69 & & \\
\hline \multirow{2}{*}{$\begin{array}{l}\text { 28. Le criticó su aspecto físico (por ejemplo, } \\
\text { ser gorda(o), delgada(o), etc.). }\end{array}$} & Rural & 80 & 0,00 & 110,50 & \multirow{2}{*}{$-2,574$} & \multirow{2}{*}{$0,010^{*}$} \\
\hline & Urbana & 152 & 0,23 & 119,66 & & \\
\hline \multirow{2}{*}{ 35. Controló su tiempo. } & Rural & 80 & 0,19 & 109,94 & \multirow{2}{*}{$-2,126$} & \multirow{2}{*}{$0,033^{*}$} \\
\hline & Urbana & 152 & 0,40 & 119,95 & & \\
\hline \multirow{2}{*}{ 44. Impuso su punto de vista sobre el suyo. } & Rural & 80 & 0,09 & 108,34 & \multirow{2}{*}{$-2,645$} & \multirow{2}{*}{$0,008^{* *}$} \\
\hline & Urbana & 152 & 0,47 & 120,80 & & \\
\hline \multirow{2}{*}{$\begin{array}{l}\text { 46. La (lo) jaló bruscamente de alguna parte del } \\
\text { cuerpo (por ejemplo, el cabello, un brazo, etc.). }\end{array}$} & Rural & 80 & 0,03 & 110,42 & \multirow{2}{*}{$-2,349$} & \multirow{2}{*}{$0,019^{*}$} \\
\hline & Urbana & 152 & 0,27 & 119,70 & & \\
\hline \multirow{2}{*}{$\begin{array}{l}\text { 63. Esculcó su bolso, maletín o sus cajones sin } \\
\text { su consentimiento }\end{array}$} & Rural & 80 & 0,06 & 110,84 & \multirow{2}{*}{$-2,064$} & \multirow{2}{*}{$0,039^{*}$} \\
\hline & Urbana & 152 & 0,29 & 119,48 & & \\
\hline \multirow{2}{*}{$\begin{array}{l}\text { 71. Le echó en cara lo que usted le había brin- } \\
\text { dado económicamente. }\end{array}$} & Rural & 80 & 0,00 & 111,50 & \multirow{2}{*}{$-2,340$} & \multirow{2}{*}{$0,019 *$} \\
\hline & Urbana & 152 & 0,19 & 119,13 & & \\
\hline \multirow{2}{*}{$\begin{array}{l}\text { 74. La (lo) llamó varias veces al día para averi- } \\
\text { guar qué estaba haciendo. }\end{array}$} & Rural & 80 & 0,38 & 108,86 & \multirow{2}{*}{$-2,021$} & \multirow{2}{*}{$0,043^{*}$} \\
\hline & Urbana & 152 & 0,70 & 120,52 & & \\
\hline
\end{tabular}

${ }^{*} p \leq 0,05^{* *} p \leq 0,01$ 
Tabla 3.

Diferencias por sexo (Kruskal-Wallis), en los tipos de conductas de maltrato ejercidas por los participantes

\begin{tabular}{|c|c|c|c|c|c|c|}
\hline Tipo de maltrato & Sexo y zona & $n$ & Mediana & Rango promedio & $x^{2}$ & $p$ \\
\hline \multirow{4}{*}{ Psicológico } & Hombre rural & 40 & 2,00 & 94,71 & \multirow{4}{*}{8,871} & \multirow{4}{*}{$0,031^{*}$} \\
\hline & Mujer rural & 40 & 3,00 & 113,89 & & \\
\hline & Hombre urbano & 72 & 5,00 & 129,55 & & \\
\hline & Mujer urbano & 78 & 2,00 & 114,02 & & \\
\hline \multirow{4}{*}{ Emocional } & Hombre rural & 40 & 0,62 & 111,08 & \multirow{4}{*}{7,916} & \multirow{4}{*}{$0,048^{*}$} \\
\hline & Mujer rural & 40 & 0,11 & 104,88 & & \\
\hline & Hombre urbano & 72 & 1,79 & 126,21 & & \\
\hline & Mujer urbano & 78 & 1,26 & 116,16 & & \\
\hline \multirow{4}{*}{ Físico } & Hombre rural & 40 & 0,38 & 105,88 & \multirow{4}{*}{4,650} & \multirow{4}{*}{0,199} \\
\hline & Mujer rural & 40 & 0,47 & 111,16 & & \\
\hline & Hombre urbano & 72 & 1,15 & 119,23 & & \\
\hline & Mujer urbano & 78 & 2,03 & 120,65 & & \\
\hline \multirow{4}{*}{ Sexual } & Hombre rural & 40 & 0,46 & 113,16 & \multirow{4}{*}{8,281} & \multirow{4}{*}{$0,041^{*}$} \\
\hline & Mujer rural & 40 & 0,42 & 119,06 & & \\
\hline & Hombre urbano & 72 & 0,69 & 125,01 & & \\
\hline & Mujer urbano & 78 & 0,32 & 109,03 & & \\
\hline \multirow{4}{*}{ General } & Hombre rural & 40 & 3,00 & 96,44 & \multirow{4}{*}{6,841} & \multirow{4}{*}{0,077} \\
\hline & Mujer rural & 40 & 4,00 & 113,70 & & \\
\hline & Hombre urbano & 72 & 7,00 & 127,91 & & \\
\hline & Mujer urbano & 78 & 2,50 & 114,74 & & \\
\hline
\end{tabular}

${ }^{*} p \leq 0,05$

\section{Discusión}

Este estudio tuvo como objetivo describir y determinar las diferencias existentes en las conductas de maltrato ejercidas en el noviazgo en adolescentes de un área rural y urbana del municipio de Tunja (Colombia). Los resultados indican que en cada sector aproximadamente la mitad de los participantes presentaron al menos una conducta de maltrato a su pareja, aunque el rango promedio de conductas de maltrato de tipo psicológico, emocional y físico, así como la frecuencia general de malos tratos, fueron significativamente mayores entre los participantes del área urbana, particularmente entre los hombres, quienes reportaron un rango promedio de conductas de maltrato psicológico, emocional y sexual significativamente mayor. Estos resultados difieren de los hallazgos del estudio de Spencer \& Bryant (2002), en el que se encontró que el porcentaje de participantes del área rural que ejerció maltrato fue mayor al de los del área urbana, así como con los resultados de la investigación de Bergman (1992), que encontró que este porcentaje era mayor entre los participantes del área urbana.

Sin embargo, en cuanto a la frecuencia de malos tratos, estos resultados tienden a señalar que esta podría ser mayor entre los(as) adolescentes de las áreas urbanas. No obstante, se requieren otros estudios que permitan dilucidar las variables que podrían estar relacionadas con las diferencias por sector en las prevalencias y las frecuencias reportadas de malos tratos ejercidos hacia la pareja en el noviazgo, como las actitudes a favor de la violencia, el consumo de sustancias, la observación de violencia en la familia de origen y la influencia de los pares (Foshee et al., 2013; McDonell et al., 2010; Montoya et al., 2013).

En cuanto a los tipos de malos tratos, los hallazgos son congruentes con los de otros estudios que indican que los malos tratos de tipo psicológico o verbal son más frecuentes que los de tipo físico 
y sexual (Rey-Anacona, 2013; Rey-Anacona et al., 2010; Muñoz-Rivas et al., 2007; Sears, Byers \& Price, 2007; Vivanco et al., 2015). Parece, entonces, que esta tendencia podría ser similar entre los(as) adolescentes de áreas rurales, quienes en este estudio reportaron una frecuencia mayor de malos tratos de tipo psicológico, que los de tipo físico y sexual (véase la tabla 1). Resultados similares fueron reportados por Rey-Anacona (2013) en una muestra urbana conformada por 902 estudiantes universitarios de 15 a 35 años de la ciudad de Tunja. Los resultados que se presentan en la tabla 2 confirman la mayor frecuencia de los malos tratos de tipo psicológico sobre los otros tipos, particularmente las conductas de control y coerción sobre la pareja, como esculcar su bolso, controlar su tiempo y llamarla constantemente, que parecen ser más aceptados entre los(as) adolescentes del área urbana que los(as) del área rural. Sin embargo, como ya se indicó, se requieren otros estudios dirigidos a examinar las variables que explicarían estas diferencias por sector.

En lo que tiene que ver con el sexo, los resultados son congruentes con los hallazgos de investigaciones previas que revelan que los malos tratos de tipo sexual son más frecuentemente ejercidos por los varones que por las mujeres (Rey-Anacona, 2013; Rey-Anacona et al., 2010; Sears et al., 2007), particularmente los del área urbana. Los malos tratos de tipo emocional (amenazas, agresiones indirectas, etc.), también se reportaron en mayor número por los varones, tanto del área rural como urbana, con una frecuencia significativamente mayor entre los de esta última zona. No obstante, la frecuencia de malos tratos de tipo físico fue mayor entre las mujeres de ambas zonas, mientras que la frecuencia general de malos tratos y los de tipo psicológico fueron mayores entre las mujeres que entre los varones del área rural, a diferencia de los(as) adolescentes del área urbana, en donde la frecuencia de malos tratos general y de tipo físico fueron reportadas en mayor número por los varones que por las mujeres. Estos resultados sugieren diferencias por área en la expresión de esta forma de violencia que amerita otras investigaciones.

En conclusión, los resultados obtenidos en este estudio, en concordancia con trabajos previos, señalan que la violencia en el noviazgo es un fenómeno que puede ser muy común entre los(as) adolescentes de las áreas rurales. Por ello, se recomienda realizar otras investigaciones que den cuenta de las prevalencias de violencia en el noviazgo en esta y en otras regiones rurales del país, con el fin de establecer las líneas de acción más apropiadas para detectar los casos entre los(as) adolescentes dentro y fuera de las instituciones educativas, diseñar e implementar programas de prevención y establecer pautas para intervenir y atender a los victimarios y las víctimas, teniendo en cuenta las diferencias socioculturales que existen entre los sectores rurales y urbanos.

Es importante señalar que los resultados de este estudio deben ser considerados como exploratorios y no pueden generalizarse debido al tipo de muestreo implementado y el uso de instrumentos de auto informe, pues muchos participantes pudieron haber reportado información falsa o imprecisa. Además, estos resultados se limitan a adolescentes de un área geográfica muy específica de Colombia, que presentaron una relación heterosexual, lo cual conlleva a la necesidad de realizar una investigación con una muestra más amplia con adolescentes con otras orientaciones sexuales en diferentes regiones del país.

\section{Referencias}

Arbach, C., Nguyen-Vo, T. y Bobbio, A. (2015). Violencia física en el noviazgo: análisis de los tipos diádicos en población argentina. Revista Argentina de Ciencia del Comportamiento, 7(2), 38-46.

Banco Mundial (2016). Población rural (\% de la población total). Recuperado de http://datos. bancomundial.org/indicador/SP.RUR.TOTL.ZS

Bergman, L. (1992). Dating violence among high school students. Social Work, 37(1), 21-27.

Borrajo, E., Gámez-Guadix, M. \& Calvete, E. (2015). Justification beliefs of violence, myths about love and cyber dating abuse. Psicothema, 27(4), 327-333. doi: 10.7334/psicothema2015.59. 
Cortés-Ayala, L., Flóres, M., Bringas, C., RodríguezFranco, L., López-Cepero, J. y Rodríguez-Díaz, F.J. (2015). Relación de maltrato en el noviazgo de jóvenes mexicanos. Análisis diferencial por sexo y nivel de estudios. Terapia Psicológica, 33(1), 5-12. doi: http://dx.doi.org/10.4067/ S0718-48082015000100001

Departamento Administrativo Nacional de Estadística (DANE) (2015a). Informe de contexto del 3er Censo Nacional Agropecuario. Recuperado de https:// www.dane.gov.co/files/CensoAgropecuario/ avanceCNA/CNA_Contexto_2015.pdf

Departamento Administrativo Nacional de Estadística (DANE) (2015b). Boyacá: pobreza monetaria 2014 (boletín técnico). Recuperado de: https://www. dane.gov.co/files/investigaciones/condiciones_ vida/pobreza/Boyaca_Pobreza_2014.pdf

Díaz, P.J. (2012). El alma colombiana. Idiosincrasia e identidades culturales en Colombia. Hallazgos, 9(18), 119-141.

Foshee, V.A., McNaughton, H.L., Gottfredson, N.C., Chang, L.Y. \& Ennett, S.T. (2013). A longitudinal examination of psychological, behavioral, academic, and relationship consequences of dating abuse victimization among a primarily rural sample of adolescents. Journal of Adolescent Health, 53, 723-729. doi: 10.1016/j. jadohealth.2013.06.016

Garrido, E.F., \& Taussig, H.N. (2013). Do parenting practices and prosocial peers moderate the association between intimate partner violence exposure and teen dating violence? Psychology of Violence, 3(4), 354-366. doi: 10.1037/a0034036

Gobernación de Boyacá (2011). Indicadores básicos en salud Boyacá 2011. Recuperado de https:// www.minsalud.gov.co/plandecenal/mapa/ Analisis-de-Situacion-Salud-Boyaca-2011.pdf

González-Ortega, I., Echeburúa, E. y Corral, P. (2008). Variables significativas en las relaciones violentas en parejas jóvenes: una revisión. Psicología Conductual, 16(2), 207-225.
Hernando, A. (2007). La prevención de la violencia de género en adolescentes. Una experiencia en el ámbito educativo. Apuntes de Psicología, 25(3), 325-340.

Marquart, B.S., Nannini, D.K., Edwards, R.W., Stanley, L.R., \& Wayman, J.C. (2007). Prevalence of dating violence and victimization: Regional and gender differences. Adolescence, 42(168), 645-657.

Martínez, J.A., Vargas, R. y Novoa, M. (2016). Relación entre la violencia en el noviazgo y observación de modelos parentales de maltrato. Psychologia: avances de la disciplina, 10(1), 101-112.

Méndez, I. y Nahimira G.D. (2000). El protocolo de investigación: Lineamientos para su elaboración y análisis. México: Trillas.

McDonell, J., Ott, J., \& Mitchell, M. (2010). Predicting dating violence victimization and perpetration among middle and high school students in a rural southern community. Children and Youth Services Review, 32, 1458-1463. doi: 10.1016/j. childyouth.2010.07.001

Montoya, T.A., Coker-Appiah, D.S., Eng, E., Wynn, M.R., \& Townsend, T.G. (2013). A qualitative exploration of rural African American youth perceptions about the effect of dating violence on sexual health. Journal of Child and Family Studies, 22, 48-62. doi: 10.1007/ s10826-012-9642-9

Muñoz, B., Ortega-Rivera, F.J. y Sánchez, V. (2013). El DaViPoP: un programa de prevención de violencia en el cortejo y las parejas adolescentes. Apuntes de Psicología, 31(2), 215-224.

Muñoz-Rivas, M.J., Gámez-Guadix, M., Graña, J.L. y Fernández, L. (2010). Violencia en el noviazgo y consumo de alcohol y drogas ilegales entre adolescentes y jóvenes españoles. Adicciones, 22(2), 125-134.

Muñoz-Rivas, M.J., Graña, J.L., O’Leary, K.D., \& González, M.P. (2007). Aggression in adolescent dating relationships: Prevalence, justification, 
and health consequences. Journal of Adolescent Health, 40(4), 298-304.

Pick, S., Leenen, L., Givaudan, M. y Prado, A. (2010). "Yo quiero, yo puedo prevenir la violencia": programa breve de sensibilización sobre violencia en el noviazgo. Salud Mental, 33(2), 153-160.

Póo, A. y Vizcarra, M. (2011). Diseño, implementación y evaluación de un programa de prevención de la violencia en el noviazgo. Terapia Psicológica, 29(2) 213-223.

Rey-Anacona, C.A. (2009). Maltrato en el noviazgo de tipo físico, psicológico, emocional, sexual y económico: un estudio exploratorio. Acta Colombiana de Psicología, 12 (2), 27-36.

Rey-Anacona, C.A. (2011). Exposición a violencia entre los padres en adolescentes y adultos jóvenes víctimas de alguna conducta de maltrato en el noviazgo. Diversitas, 7(2), 253-264.

Rey-Anacona, C.A. (2013). Prevalencia y tipos de maltrato en el noviazgo en adolescentes y adultos jóvenes. Terapia Psicológica, 31(2), 143-154.

Rey-Anacona, C.A. (2015). Variables asociadas a los malos tratos en el noviazgo en adolescentes y adultos jóvenes. Acta Colombiana de Psicología, 18(1), 159-171.

Rey Anacona, C.A., Mateus Cubides, A.M. y Bayona Arévalo, P.A. (2010). Malos tratos ejercidos por adolescentes durante el noviazgo: diferencias por sexo. Revista Mexicana de Psicología, 27(2), 169-181.

Rivera, L., Allen, B., Rodríguez, G., Chávez, R. \& Lazcano, E. (2007). Prevalence and correlates of adolescent dating violence: Baseline study of a cohort of 7960 male and female Mexican public school students. Preventive Medicine, 44(6), 477-484.

Saldivia, C. y Vizcarra, B. (2012). Consumo de drogas y violencia en el noviazgo en estudiantes universitarios del sur de Chile. Terapia Psicológica, 30(2), 43-49. doi: 10.4067/ S0718-48082012000200004

Sears, H.A., Byers, E.S., \& Price, E.L. (2007). The co-occurrence of adolescent boys' and girls' use of psychologically, physically, and sexually abusive behaviours in their dating relationships. Journal of Adolescence, 30(3), 487-504. doi: 10.1016/j.adolescence.2006.05.002

Spencer, G.A., \& Bryant, S.A. (2002). Dating violence: A comparison of rural, suburban, and urban teens. Journal of Adolescent Health, 27(5), 302-305.

Vivanco, R., Espinoza, S., Romo, C., Véliz, A. y Vargas, A. (2015). Perpetración y victimización de la violencia en relaciones de parejas en jóvenes que cursan educación superior en la ciudad de Osorno, Chile. Polis Revista Latinoamericana, 40, 1-15. 